\title{
INTEGRATED THERMAL PROCESS OPTIMIZATION OF ALLOY 718PLUS® FOR ADDITIVE MANUFACTURING
}

\author{
Jiadong Gong ${ }^{1}$, Hallee Z. Deutchman ${ }^{2}$, Alonso Peralta ${ }^{2}$, Dave Snyder ${ }^{1}$, Michael P. Enright ${ }^{3}$, John McFarland ${ }^{3}$, James Neumann ${ }^{2}$, Jason \\ Sebastian ${ }^{1}$, Greg Olson ${ }^{1}$ \\ ${ }^{1}$ QuesTek Innovations; 1820 Ridge Avenue; Evanston, IL, 60201, USA \\ ${ }^{2}$ Honeywell Aerospace; 111 S. $34^{\text {th }}$ Street; Phoenix, AZ, 85034, USA \\ ${ }^{3}$ Southwest Research Institute; 6220 Culebra Road; San Antonio, TX, 28510, USA
}

Keywords: 718Plus, microstructure, additive manufacturing, ICME

\section{Introduction}

Honeywell Aerospace's DARPA Open Manufacturing program (DARPA OM) is a combined effort of integrated computational materials engineering (ICME) models and mindset enabling a rapid qualification of components for the aerospace industry. The program focuses on laser beam additive manufacturing of Nickelbased Alloy 718Plus, emphasizing an integrated empirical and modeling effort for prediction of material properties produced by this novel process. As this emerging additive manufacturing technology matures, it has forced tools to model and simulate the intricate process to advance to a new level. The complexity during the additive process, such as the rapid heating rates, cooling rates, and transient phase transformation, brings increased uncertainty to the microstructure and material properties of the components. The post build processing for ATI 718Plus ${ }^{\circledR}$ Alloy used in this program includes a Stress Relief (SR) followed by Hot Isostatic Pressure (HIP) cycle, and then Solution Heat Treatment (SHT) followed by a two-step Aging. However, the standard post treatment that has been developed for the traditionally formed material often falls short for the additively processed material. This program focuses on mining empirical data to build the next generation of models, and drive future rapid qualification of aerospace components.

This paper will discuss the unique microstructure evolution that results from additive manufacturing, how the DARPA team calibrated and validated ICME models for that evolution and for strength modeling, and how the resulting models assisted the development of a new optimized stress-relief temperature.

\section{Material and Additive Processing}

In short, additive manufacturing is a method that takes powder particles of specific size and melts them in accordance with a 3D model or CAD file. While additive is exciting for many in the manufacturing realm, additive is also opening up completely new ways of processing and understanding previously well characterized materials. The thing that makes additive manufacturing unique as far as processing is the extreme cooling rates the materials experience, which are on the order of $10^{6}$ degrees per second. These extreme cooling rates produce columnar grains (aided by the preferential cooling towards the plate) with fine microstructural features, as well as the possibility for defects such as process-induced porosity or cracking.

During this program, several build conditions were performed in search of fully dense material on an EOS M280 laser powder bed machine at Honeywell Aerospace. After several major DOE's, the following machine parameters were deemed to be "optimal" for 718 Plus with minimal porosity in a DMLS or laser-based additive manufacturing (also known as "DMLS") were: 195 W laser power, hatch spacing $0.100 \mathrm{~mm}$, laser speed $1000 \mathrm{~mm} / \mathrm{second}$, and stripe width of $5 \mathrm{~mm}$.

The samples described in this paper were all built using these parameters, in several different geometries to answer questions about specific parts of the program. For example, for the heat treatment investigation, small cubes were built. For the tensile testing, tall cylinders were additively manufactured and then machined down to the desired dimensions.

\section{Microstructure Evolution}

ICME models have been developed and calibrated to predict microstructure and strength properties of 718Plus components produced using laser bed additive manufacturing. These models developed during this program predicts the microstructural evolution of the material during the heat treatment process.

The standard post build processing used for this program includes a SR cycle at $1065{ }^{\circ} \mathrm{C}\left(1950{ }^{\circ} \mathrm{F}\right)$ for 1.5 hours; followed by HIP cycle at $1160^{\circ} \mathrm{C}\left(2125^{\circ} \mathrm{F}\right)$ and $103 \mathrm{MPa}(15 \mathrm{ksi})$ for 4 hours, then SHT at $970{ }^{\circ} \mathrm{C}\left(1775^{\circ} \mathrm{F}\right)$ for 2 hours, followed by a two-step age at $790{ }^{\circ} \mathrm{C}\left(1450{ }^{\circ} \mathrm{F}\right)$ and $700{ }^{\circ} \mathrm{C}\left(1300{ }^{\circ} \mathrm{F}\right)$ for 8 hours each. The prediction of the transient microstructure evolution during the DMLS build is not yet being pursued in this program. Instead, an effective HIP cycle is sought so that the material can be fully homogenized after the HIP cycle and that the microstructure of the final part would depend exclusively on the subsequent heat treatment.

While the experimental method provided direct evidence of the microstructure under various processing conditions, the ICME analytical approach was used to predict the microstructure, then compared with the experimental data to perform a calibration of the models. The intent is to show how the concurrent use of the analytical models and the experimental effort yield a much more robust approach for qualification than the classical approach given that a calibrated and validated analytical model can be used to explore, in a much more efficient way, any variety of post processing conditions to understand the effects of varying conditions on microstructure and property variation.

Beginning with the experimental inputs, a full microstructural assessment of 718Plus processed by DMLS was conducted to characterize the development of microstructures through the complex thermal processing route. Samples were characterized at each of the stages of the heat treatment cycle using standard metallographic techniques. Light optical microscopy at low magnifications was used to observe the distinct as-solidified melt pool regions of the numerous laser tracks. Increasing magnifications reveal the fine dendritic structure of the 
solidification segregation. SEM was used to observe several of these phases and verify their constitution.

Figure 1 contains example micrographs of the as-built 718Plus microstructure over a range of magnifications. At low magnifications (A), distinct as-solidified melt pool regions of the numerous laser tracks can be seen. Increasing magnifications reveal the fine dendritic structure of the solidification segregation. High resolution SEM is needed to observe the highly refined secondary dendrite arm spacing, which was measured at an average of $0.4 \mu \mathrm{m}$. The measured Secondary Dendrite Arm Spacing (SDAS) was later used to estimate the cooling rate during the solidification [1], which was fed back into the simulations.

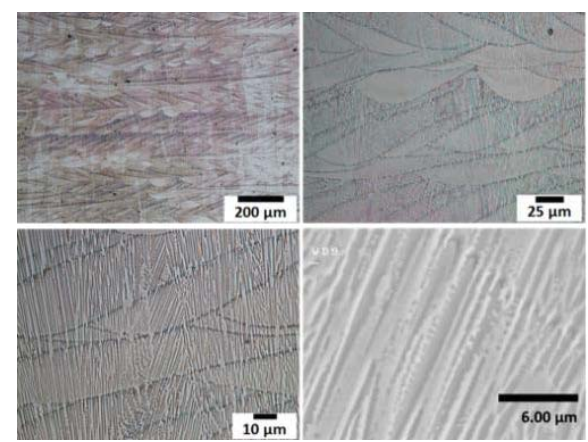

Figure 1. A-C) Light optical micrographs of the As-built 718 Plus microstructure at various magnifications, showing the melt pool regions and aligned grain and dendritic structures (etched in Glycergia). D) SEM magnification of the dendritric structure
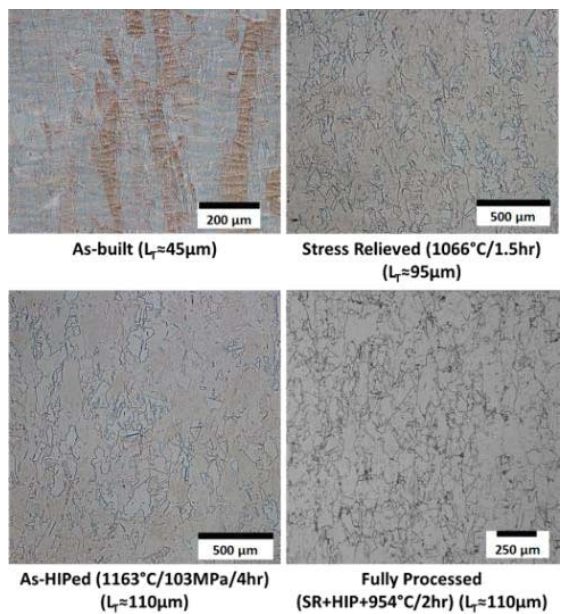

Figure 2. Microstructure and grain size evolution

Since as built grains are columnar in shape, the first stress relief process was found to be critical for the as-built microstructure recrystallizing to an equiaxed microstructure. Figure 2 contains example micrographs of the evolution in microstructure through the sequence of thermal treatments from as-built, stress relieved, SR + HIP, and SR + HIP + solution treated. It was determined that stress relieving at $1065{ }^{\circ} \mathrm{C} \quad\left(1950{ }^{\circ} \mathrm{F}\right)(2 \mathrm{~b})$ results in complete recrystallization of the as-built structure. The equiaxed grain structure is maintained through further processing at lower temperature. As shown in Figure 2, a minor change in average grain size accompanies the additional processing steps beyond this initial stress relief. The HIP cycle has been identified as the dominant processing step determining grain structure since it requires the highest temperature. The final linear intercept grain size is determined to be $110 \mu \mathrm{m}$.

Grain structure evolution during the whole process was also characterized with scanning electron microscopy and the stress relaxation and recrystallization during the post process has been quantified with electron backscattered diffraction (EBSD). There are two primary methods of quantifying lattice strain by EBSD [24]:

1) EBSD pattern quality approach. This approach utilizes metrics of pattern quality to quantify the diffraction peak broadening that results from lattice strain. A primary metric used is band contrast, which is a relative ranking of the diffraction pattern "sharpness". Increased lattice strain is accompanied by decreasing band contrast.

2) Local lattice misorientation approach. This approach quantifies lattice strains by the local lattice rotations that accompany dislocations and dislocation substructures. Analysis is typically conducted on intragranular misorientations to exclude misorientations associated with grain boundaries. Grains are subdivided into finite spacial volumes (i.e. $50 \mathrm{~nm} \times 50 \mathrm{~nm}$ square pixels), and lattice orientation is measured at each pixel. Lattice misorientations are then calculated as the angle of misorientation between each adjacent pixel, and a map of lattice misorientation is generated across each grain. Typically, the mean intragranular misorientation (MIM) is quantified as the average misorientation measured from the mean grain orientation. This is measured for each grain individually to quantify the spread in intragranular misorientations across a broad sampling of grains, then averaged across the entire map to yield a single metric of overall MIM.

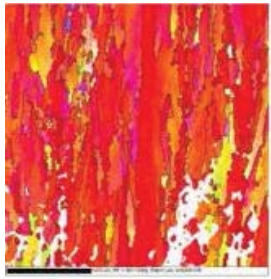

(A)

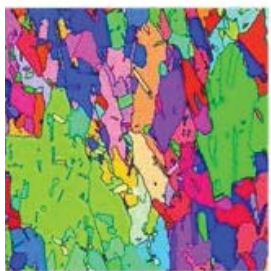

(D)

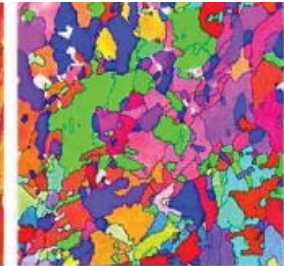

(B)

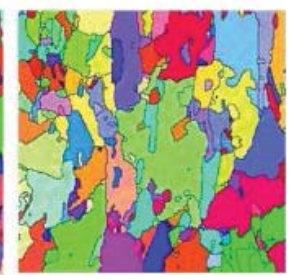

(E)

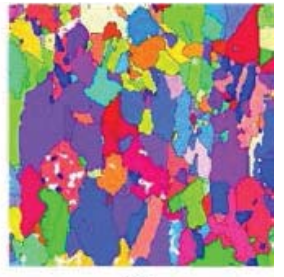

(c)

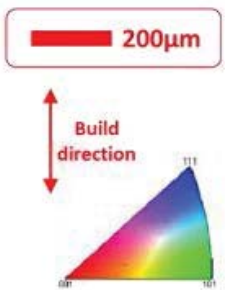

Figure 3. EBSD maps of the microstructures of 718Plus DMLS in the A) As-built, B) Stress-relieved, C) Stress Relieved and HIP, D) Stress Relieved, HIP and $950^{\circ} \mathrm{C}\left(1750^{\circ} \mathrm{F}\right)$ Solution treated, and E) Stress Relieved, HIP and $1065^{\circ} \mathrm{C}\left(1950^{\circ} \mathrm{F}\right)$ solution treated conditions.

Figure 3 shows EBSD images of the microstructure for various processing conditions, showing grain orientation relative to the build direction. The fully processed alloy 718Plus possesses a complex microstructure with multiple phases present. SEM was used to observe several of these phases and verify their constitution. Figures 4-6 show representative micrographs of the $\delta$ phase, 
carbide, and oxide phases present in the alloy. Corresponding EDS spectral analyses were performed to validate their constitution. Carbide phases were identified to be primarily $\mathrm{Nb}$-based, with a small amount of Ti. Oxide phases were found to be primarily Albased, also with a minor amount of Ti.

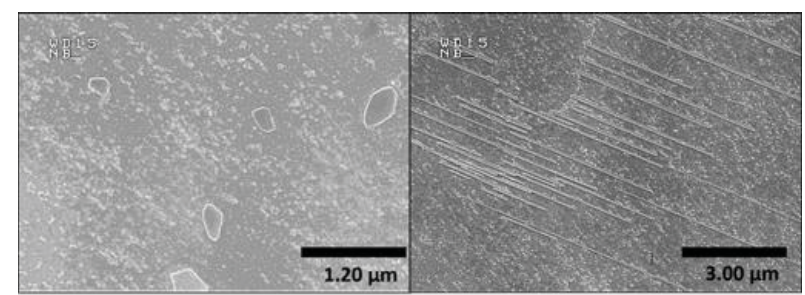

Figure 4. SEM images of primary $\delta$-phase particles (both platelike and equiaxed) in the fully processed 718Plus material

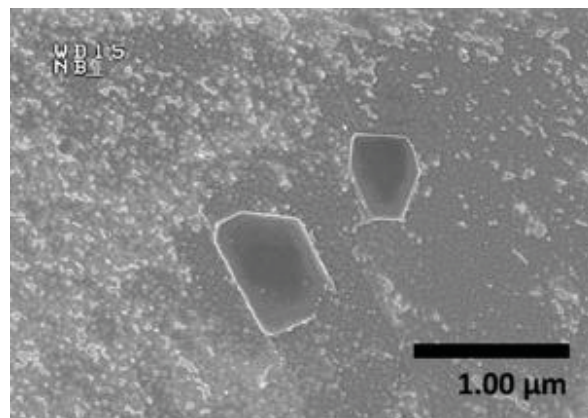

Figure 5. SEM image of ( $\mathrm{Nb}$, Ti) C carbide particles in the fully processed 718 Plus material

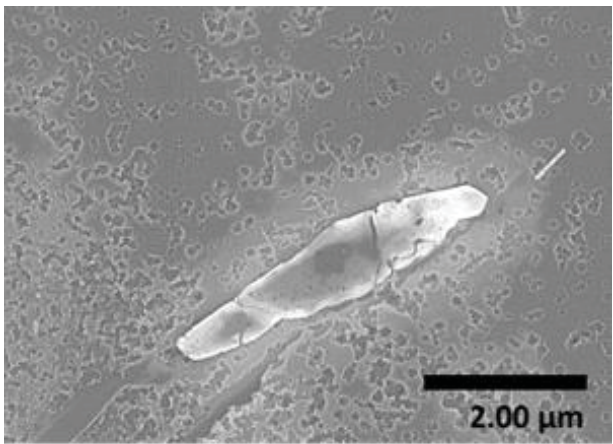

Figure 6. SEM image of $\mathrm{Al}_{2} \mathrm{O}_{3}$ oxide particles in the fully processed 718 Plus material.

Figure 7 shows the $\gamma^{\prime}$ precipitates revealed by high resolution SEM in the fully processed 718Plus DMLS material after double-step aging. The average radius of the $\gamma^{\prime}$ precipitates is estimated to be 14 $+/-3 \mathrm{~nm}$.
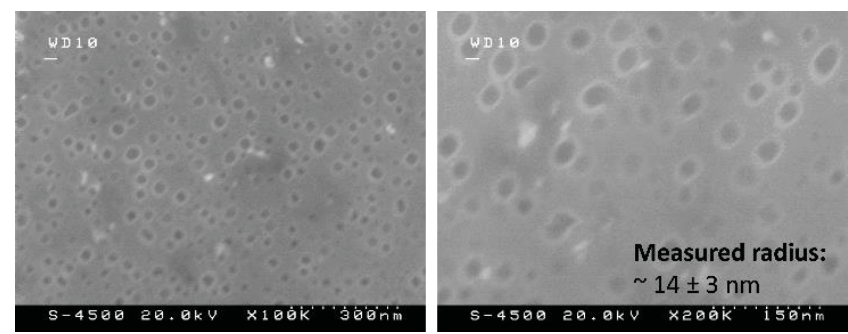

Figure 7. High-resolution SEM micrographs of the $\gamma^{\prime}$ precipitates in the fully processed 718Plus DMLS material (double-step aged). Etched with Glycergia.

\section{Thermodynamic Modeling}

CALPHAD predictions of phase constitution employed the commercial thermodynamic database (Thermotek NiData V.7 and ThemoCalc TCNI6) have been completed. The equilibrium step diagram was computed to show the phase fraction of the primary phases, as well as the oxide $\left(\mathrm{Al}_{2} \mathrm{O}_{3}\right)$, nitride (TiN), boride $\left(\mathrm{Mo}_{2} \mathrm{CrB}_{2}\right)$ and carbides $(\mathrm{NbC})$, in Figure 9. With the current powder composition, the solution treatment temperature commonly used in wrought 718Plus lies in the subsolvus range of the $\delta-\mathrm{Ni}_{3} \mathrm{Nb}$. Primary $\gamma^{\prime}$ as well as the secondary $\gamma^{\prime}$ fractions for different heat treatment conditions have also been computed.

Solidification microsegregation and HIP homogenization simulation has also been completed using Scheil model and DICTRA simulation (Figure 9), predicting rapid homogenization with the extremely fine secondary dendrite structures obtained.

Aging treatment simulation was performed using QuesTek's PrecipiCalc ${ }^{\circledR}$ modeling tool to predict the particle size, number density, volume fraction of the $\gamma^{\prime}$, and precipitate size distribution during the two-step aging, as shown in Figure 8. The predicted final $\gamma^{\prime}$ precipitate radius $(\sim 14.6 \mathrm{~nm})$ agrees very well with literature data from TEM and LEAP in wrought 718Plus and SEM measurement of DMLS processed 718Plus (Table I). The compositions of the $\gamma^{\prime}$ precipitate and the matrix have also been calculated, and comparison with LEAP data shows a RMS composition error less than $1 \%$.
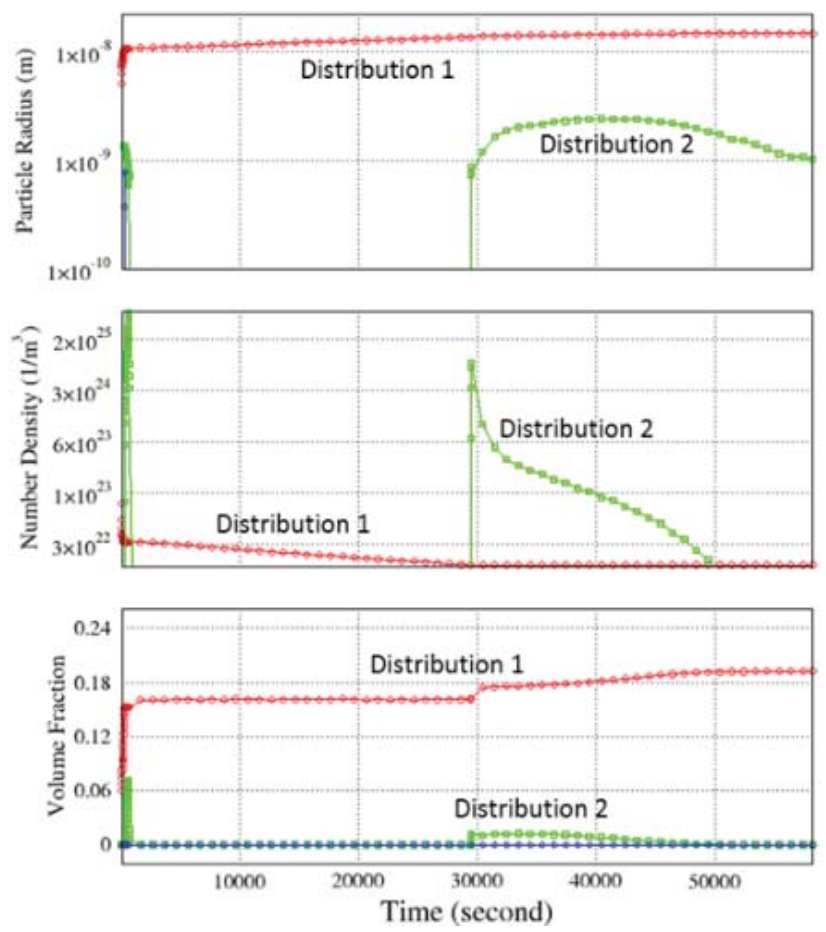

Figure 8. PrecipiCalc ${ }^{\circledR}$ prediction of the particle size, number density, volume fraction of the $\gamma^{\prime}$, and size distribution during the two-step aging. 

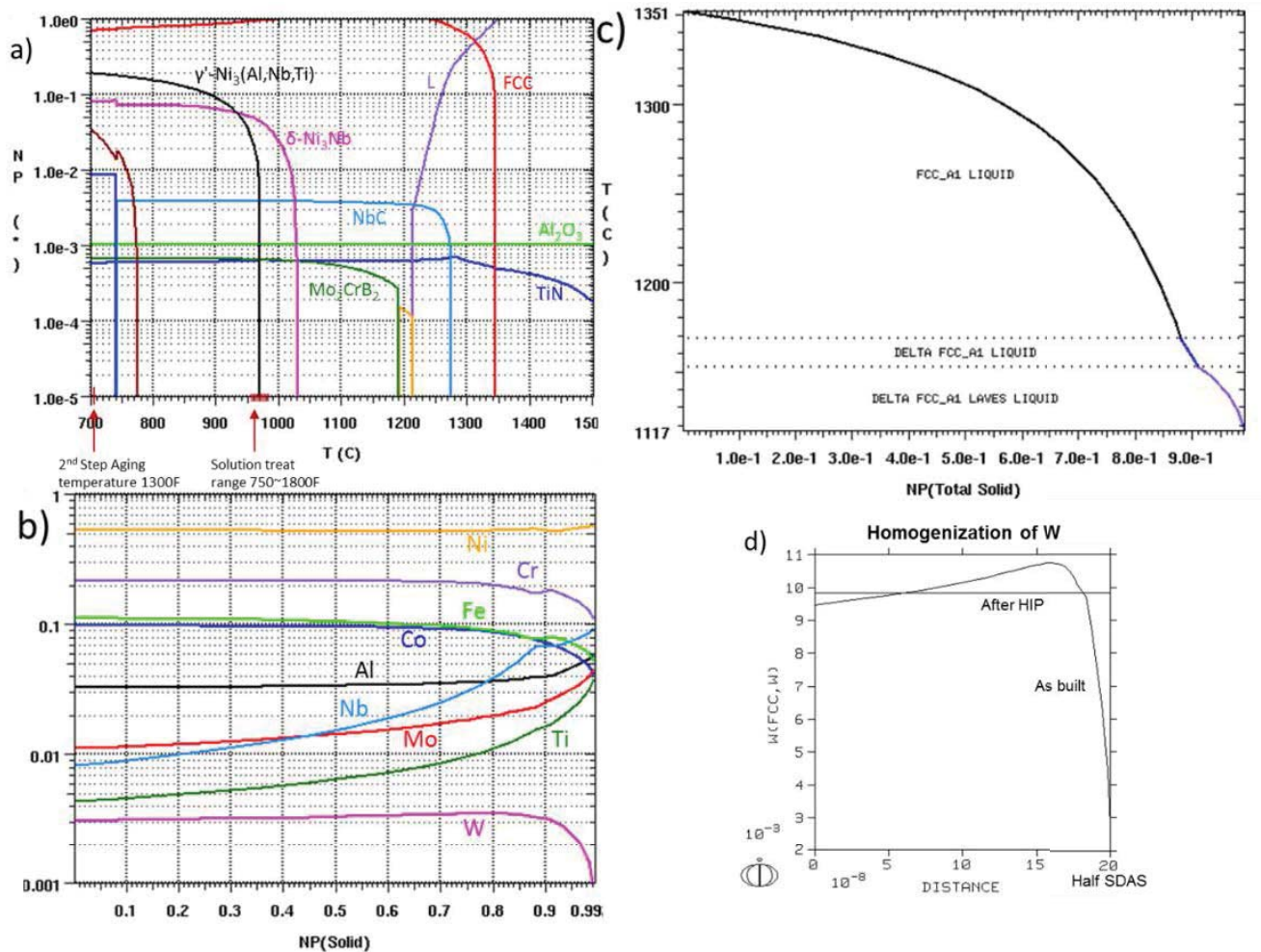

Figure 9. a) Equilibrium step diagram of 718Plus with standard heat treatment temperatures marked; b) Scheil simulation of solidification; c) Scheil microsegregation profiles; and d) Homogenization during the HIP (W as an example).

Table I. Comparison of the prediction compositions with the results measured from LEAP.

\begin{tabular}{|c|c|c|c|c|c|c|c|c|c|c|c|}
\hline \multicolumn{2}{|c|}{ Composition in at $\%$} & $\mathrm{Ni}$ & $\mathrm{Cr}$ & $\mathrm{Fe}$ & $\mathrm{Co}$ & Mo & $\mathrm{Al}$ & W & $\mathrm{Nb}$ & $\mathrm{Ti}$ & RMS \\
\hline \multirow{2}{*}{ matrix } & prediction & 45.65 & 26.92 & 12.80 & 10.79 & 2.17 & 0.92 & 0.36 & 0.35 & 0.03 & \multirow{2}{*}{0.66} \\
\hline & LEAP & 45.33 & 26.44 & 12.40 & 11.50 & 2.11 & 0.46 & 0.40 & 1.30 & 0.10 & \\
\hline \multirow{2}{*}{$\gamma^{\prime}$} & prediction & 70.47 & 1.13 & 1.27 & 2.88 & 0.20 & 11.91 & 0.21 & 8.48 & 3.44 & \multirow{2}{*}{0.37} \\
\hline & LEAP & 70.60 & 0.75 & 1.27 & 3.17 & - & 11.33 & - & 8.65 & 3.20 & \\
\hline
\end{tabular}

\section{Optimization for Material Strength}

Since additively manufactured materials are produced with an extreme cooling rate, the unusual as-built structure has necessitated previously standard heat treatments to be re-affirmed, and in some cases, changed to accommodate the different structure. The goal is usually to meet or exceed the properties of the traditionally cast (or even wrought) material.

Comparing the experimental strength results with available data, the additively manufactured 718Plus has a strength level between the cast and the wrought counterparts, consistent with the different grain sizes produced by the three methods (Figure 10). The improvement over the casting process will allow for the integration of additively manufactured components in the near future. To enable that, an accurate strength model needs to be established and calibrated to the additively manufactured 718Plus, for both room temperature and elevated temperature service.

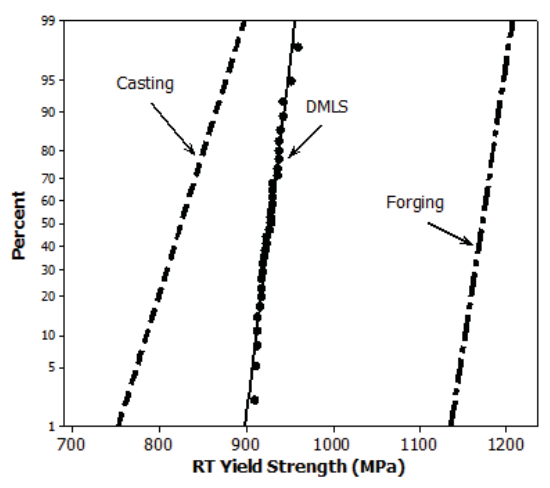

Figure 10. Comparison of Experimental 718Plus yield strength data for DMLS with casting and forging properties

\section{Strength Modeling}

Room temperature tensile tests were run with bars that had been additively manufactured in three different build orientations, to begin to understand mechanical properties and calibrate a yield 
strength model. Standard heat treatments were applied following stress relief at $1065^{\circ} \mathrm{C}\left(1950{ }^{\circ} \mathrm{F}\right)$ and HIP at $1160{ }^{\circ} \mathrm{C}\left(2125^{\circ} \mathrm{F}\right)$, corresponding to the coarse equiaxed grain structure of Figure 3E. Bars were taken from three areas to assess variation; excised from an aerospace component, built concurrently as bars with the component, and built separately from the component. It was determined that bars built vertically had a lower average yield strength Figure 11) than the other two groups, mostly due to grain size variation. These $0.2 \%$ yield strength values were also used as inputs to calibrate the DARPA AIM yield strength model [5] to 718 Plus.

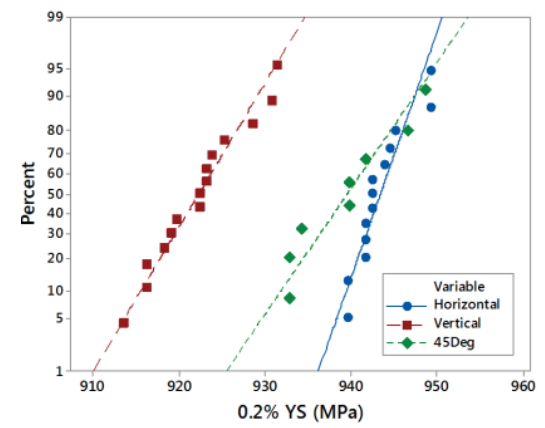

Figure 11. Probability plot of $0.2 \%$ yield strength of additively manufactured bars built in three directions

Microstructural evaluation of this material allowed for the strengthening contributions of the primary phases to be quantified and modeled. The most significant strengthening contribution, the precipitation strengthening of the secondary $\gamma^{\prime}$, has been modeled and calibrated, where anti-phase boundary (APB) energy was identified as the highest sensitivity factor. The microstructure of the 718Plus alloy consists of a mainly $\gamma / \gamma^{\prime}$ structure, with the $\gamma^{\prime}$ precipitate as the principal strengthening phase. The $\delta$ phase is also observed after subsolvus solution treatments. To model the strength of 718Plus, the detailed multiphase strengthening model developed by Pollock, Milligan, and coworkers $[5,6]$ under the DARPA-AIM program was extended to incorporate a contribution from a primary $\delta$ phase. In the subsolvus solution treated material, the strength from the contribution of $\gamma$, primary $\gamma^{\prime}$, and $\delta$ phases can then be written as:

$\sigma_{\text {yield }}\left(D_{\text {gr }} ; \mathbf{f}_{\gamma} ; \mathbf{f}_{\gamma_{p}^{\prime}} ; \mathbf{f}_{\delta}\right)=\mathbf{f}_{\gamma} \boldsymbol{\sigma}^{\gamma}+\mathbf{f}_{\gamma_{\mathbf{p}}^{\prime}} \boldsymbol{\sigma}^{\gamma_{p}^{\prime}}+\mathbf{f}_{\delta} \boldsymbol{\sigma}^{\delta}$

Here $f$ represents the phase fraction such that: $f_{\gamma}+f_{\delta}+f_{\gamma_{p}^{\prime}}=1$.

Starting with estimating the strength of the $\gamma$ matrix, contributions from the Hall-Petch grain size effect and solid solution strengthening mechanisms are considered. The strength of the $\gamma$ matrix is thus expressed as:

$\sigma^{\gamma}=\sigma^{\mathrm{HP}}+\Delta \boldsymbol{\sigma}^{\mathrm{SS}}$

The functional form for the Hall-Petch strengthening mechanism is given as

$\sigma^{\mathrm{HP}}=\frac{\mathbf{K}^{\mathrm{HP}}}{\sqrt{\mathbf{D}_{\mathrm{gr}}}}$

An estimate of the Hall-Petch coefficient $\left(K^{H P}\right)$ from existing literature on $\gamma / \gamma^{\prime}$ nickel-based superalloys gives a value of $628 \mathrm{MPa}$ $\sqrt{\mu m}$.

Solid solution is given as:
$\Delta \boldsymbol{\sigma}^{S S}=\left(\sum_{\mathbf{i}}\left(\mathbf{k}_{\mathbf{i}}\right)^{\mathbf{n}} \mathbf{C}_{\mathbf{i}}\right)^{1 / n}$

, where $k_{i}$ is the strengthening coefficient of element $i, C_{i}$ is the atomic fraction of solute element $i$ in the $\gamma$ matrix, and $n=2$. [7]

As in ref [6] strengthening coefficients were taken by Roth et al., who calculated it using experimental data at $77 \mathrm{~K}$ from Mishima's work $[8,9]$. The strengthening coefficients are taken as temperature dependent according to Roth's analysis.

Based on this approach proposed above the solution strengthening of the $\gamma$ phase at room temperature is estimated to be $256 \mathrm{MPa}$.

The strengthening of the primary $\gamma^{\prime}$ precipitates is modeled as:

$\sigma^{\gamma_{p}^{\prime}}=\sigma_{0}^{N_{3} A l}+\Delta \sigma^{S S}$

Here $\sigma_{0}^{N i_{3} A l}$ is the 'base strength' of the $\gamma$ ' $\left(\mathrm{Ni}_{3} \mathrm{Al}\right)$ phase, estimated from Kozar et al., as $100 \mathrm{MPa}$ at room temperature.

The solution hardening of $\gamma^{\prime}$ is described in Kozar et al. as

$\Delta \boldsymbol{\sigma}^{S S}=\sum_{\mathbf{i}}\left(\frac{d \sigma}{\mathrm{dC}_{\mathrm{i}}} \mathbf{C}_{\mathbf{i}}\right)$

Where $\frac{\mathbf{d} \boldsymbol{\sigma}}{\mathbf{d C}_{\mathbf{i}}}$ is the rate of hardness increase due to an element and $\mathbf{C}_{\mathbf{i}}$ is the concentration of the solute in atomic $\%$.

Based on the coefficients employed by Kozar et al [6], the contribution of the solid solution strengthening to the $\gamma^{\prime}$ phase is $158 \mathrm{MPa}(23 \mathrm{ksi})$ with the total strength of the $\gamma^{\prime}$ phase estimated to be $258 \mathrm{MPa}(37 \mathrm{ksi})$.

To estimate the strength of the $\delta$ phase, the measured strength of the as quenched material and the strength estimates of the $\gamma / \gamma^{\prime}$ phases in conjunction with equation 2 was used. The strength of the as quenched material is estimated from hardness measurement using nano indentation experiments.

Aging treatment introduces the additional precipitation strengthening of secondary $\gamma^{\prime}$. Since the secondary $\gamma^{\prime}$ forms in the $\gamma$ matrix we describe the precipitation strengthening effects as an increase of matrix strength. The yield strength of the material is described by modifying equation 2 as:

$$
\begin{aligned}
\sigma_{\text {yield }}\left(D_{\text {gr }} ; f_{\gamma} ; f_{\gamma_{p}^{\prime}} ; f_{\delta} ; f_{\gamma_{s}^{\prime}} ; r ; \gamma_{A P B}\right) \\
=\mathrm{f}_{\left(\gamma+\gamma_{\mathrm{s}}^{\prime}\right)} \sigma^{\left(\gamma+\gamma_{\mathrm{s}}^{\prime}\right)}+\mathrm{f}_{\gamma_{\mathrm{p}}^{\prime}} \sigma^{\gamma_{\mathrm{p}}^{\prime}}+\mathrm{f}_{\delta} \sigma^{\delta}
\end{aligned}
$$

where $f_{\left(\gamma+\gamma_{s}^{\prime}\right)}+f_{\delta}+f_{\gamma_{p}^{\prime}}=1$.

The strength of the matrix including the precipitation strengthening effects is given by:

$\sigma^{\left(\gamma+\gamma_{\mathrm{s}}^{\prime}\right)}=\sigma^{\mathrm{HP}}\left(\mathrm{D}_{\mathrm{gr}}\right)+\boldsymbol{\sigma}^{\mathrm{SS}}\left(\mathrm{C}_{\mathrm{i}}\right)+$
$\boldsymbol{\sigma}^{\operatorname{Precip}}\left(\mathbf{f}_{\gamma_{\mathrm{s}}^{\prime}} ; \mathbf{r} ; \boldsymbol{\gamma}_{\mathrm{APB}}\right)$

The particle strengthening theory for nickel based $\gamma / \gamma^{\prime}$ systems as described by Reppich $[10,11]$ divides the strengthening behavior into three separate regions dependent on particle size: weakly coupled dislocation shearing, strongly coupled shearing, and dislocation bypass. The equations and coefficients adapted here to describe these competing mechanisms are presented in the original DARPA-AIM strength model $[5,6]$.

The predicted variation of the critical shear stress required to operate different dislocation shearing mechanisms can then be treated as a function of particle size. Note that the optimum size of 
the precipitate occurs during the transition from weakly coupled dislocation shearing to strongly coupled dislocation shearing at a particle size of $14 \mathrm{~nm}$. This is in excellent agreement with the precipitate sizes predicted by PrecipiCalc ${ }^{\circledR}(13 \mathrm{~nm})$ and observed in the 718Plus alloy for the standard peak strength aging treatment.

Using these precipitate strengthening mechanisms, the strength increment to the matrix and subsequently the alloy is expressed as equations (7) and (8). A value of $266 \mathrm{~mJ} / \mathrm{m}^{2}$ has been used for the anti-phase boundary energy and the volume fraction of the secondary $\gamma^{\prime}$ is $17.8 \%$ (from PrecipiCalc $\AA$ ). Using these values, and the strength model described in equation (7), the predicted value of the yield strength for the material solution treated at 970 ${ }^{\circ} \mathrm{C}\left(1775^{\circ} \mathrm{F}\right)$ and double aged is $960 \mathrm{MPa}(140 \mathrm{ksi})$. Experimental values for similarly processed materials range from 911 to 1000 $\mathrm{MPa}$ (132 to $145 \mathrm{ksi}$ ) as shown in Figure 12, which shows a comparison of the predictions of the yield strength model with calibration, note that although the mean value is predicted well, a calibration must also be performed to match the scatter of the experimental data. In this way, uncertainty analysis and AIM minimum property forecast has been demonstrated in comparison with traditional statistical methods in order to give a statistically significant comparison.

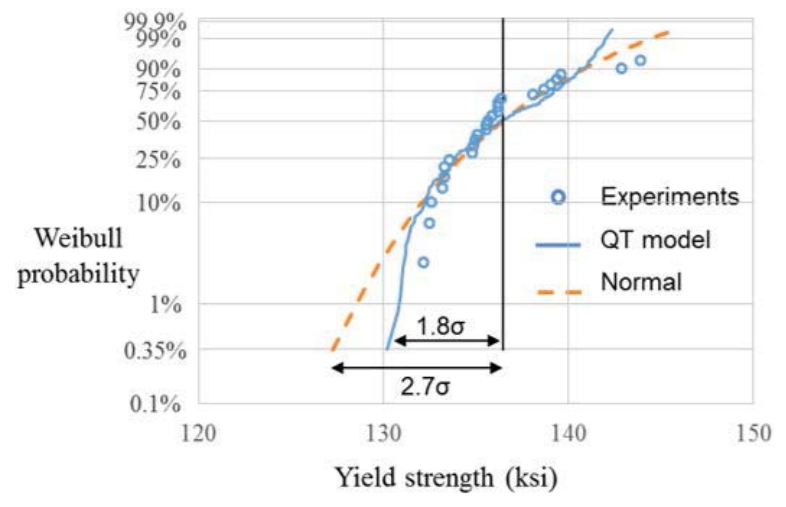

Figure 12. Minimum strength forecast for Alloy 718 Plus

\section{Temperature Dependence of Strength}

The temperature dependence of APB energy is calculated by a QuesTek thermodynamic model combing model of Saunders \& Miodownik [12] and Sluter [13]. Figure 13 compares results using two databases, ThermoTek NI7 and ThermoCalc TCNI6.

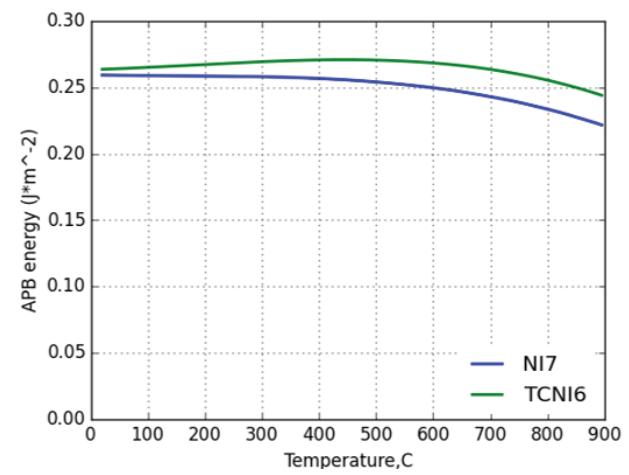

Figure 13. Predicted APB energy variation with temperature in 718 Plus
Therefore, the overall strength dependence on in 718Plus is predicted using equation (8) with results shown in Figure 14 for wrought 718 Plus. It is evident that precipitation strengthening is the most important mechanism in 718Plus limited by predicted $\gamma^{\prime}$ coarsening above $700{ }^{\circ} \mathrm{C}$.

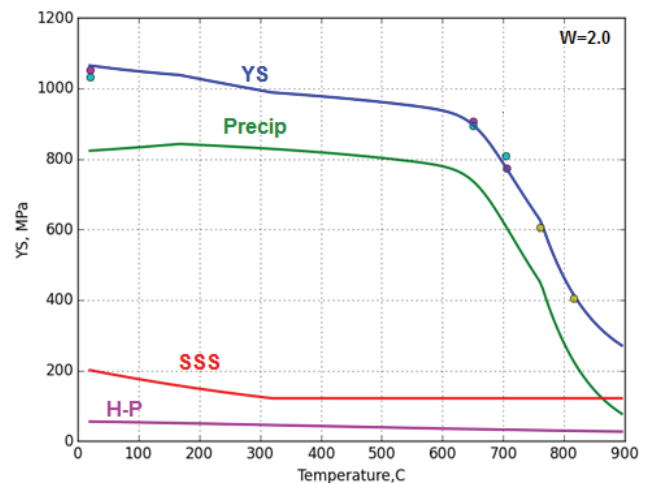

Figure 14. Overall yield strength and contributions of other mechanisms in wrought 718Plus. Dots are literature data.

\section{Ultimate Tensile Strength}

The modeling of the ultimate strength of the additively manufactured material is based on the validated yield strength prediction and a DARPA-AIM strain hardening model [14] calibrated to experiment data. The ultimate strength can be predicted from the necking criterion, $\sigma_{t}=\frac{d \sigma_{t}}{d \varepsilon_{t}}$. Using a simplified strain hardening model of the form:

$$
\sigma_{t}=\sigma_{0}\left(1+K \varepsilon_{p}^{n}\right)
$$

The calibrated result is shown in Figure 16, where the crossing of the red curve and blue curve representing the prediction of the UTS. The dots near the crossing are the measured UTS points of multiple tensile tests and the dots near the end of the red curves representing fracture. It is shown that UTS prediction is well in the range of measurement.

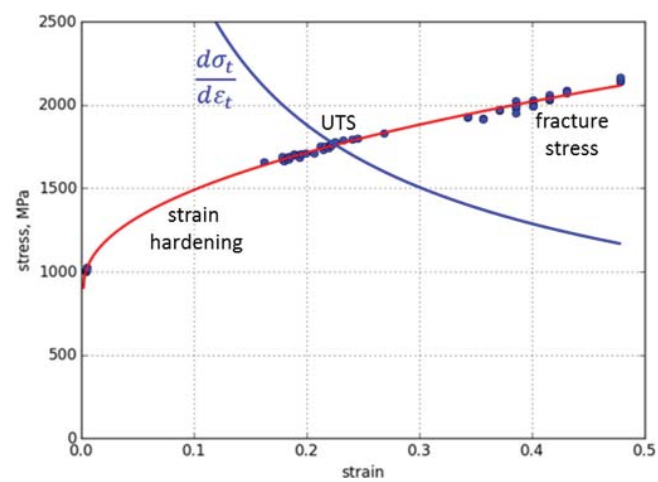

Figure 15. Ultimate strength prediction using a DARPA-AIM strain hardening model calibrated to experiment data

\section{Thermal Process Optimization}

It is already shown that grain structure differences are the main factors that affect the strength. To optimize the thermal process of DMLS Alloy 718Plus, special attention is paid to the development of microstrain with annealing temperature, in order to identify the minimum temperature necessary for full stress relief while achieving full recrystallization. Samples of as-built 718Plus were 
prepared using the previously identified optimal minimum porosity conditions.

Samples were sectioned into individual test coupons nominally $3 \mathrm{~mm}$ (length) by $4 \mathrm{~mm} \times 5 \mathrm{~mm}$. Heat treatments were conducted isochronally (fixed 1.5 hours in duration) at $950{ }^{\circ} \mathrm{C}\left(1750^{\circ} \mathrm{F}\right), 980$ ${ }^{\circ} \mathrm{C}\left(1800^{\circ} \mathrm{F}\right), 1010^{\circ} \mathrm{C}\left(1850{ }^{\circ} \mathrm{F}\right), 1030^{\circ} \mathrm{C}\left(1900{ }^{\circ} \mathrm{F}\right)$ and $1065^{\circ} \mathrm{C}$ $\left(1950^{\circ} \mathrm{F}\right)$ (the current stress relief cycle experimentally validated to effectively relieve stored strain). This temperature range was selected to bond conditions previously identified to yield no recrystallization of the material $\left(1750^{\circ} \mathrm{F}\right)$ and full recrystallization $\left(1065^{\circ} \mathrm{C}\right.$ or $\left.1950^{\circ} \mathrm{F}\right)$. The as-built material was also analyzed as a baseline condition. Samples were prepared by standard metallographic methods and characterized by EBSD using the scanning electron microscopy facilities at Northwestern University (Evanston, IL).
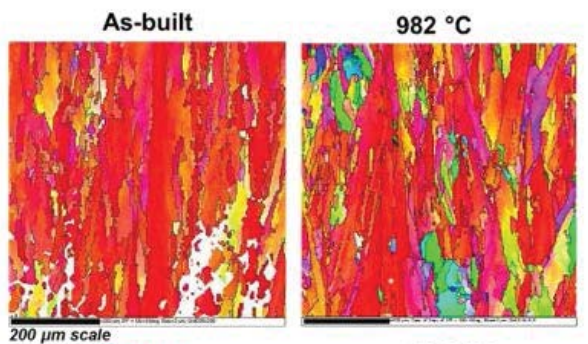

$1010^{\circ} \mathrm{C}$
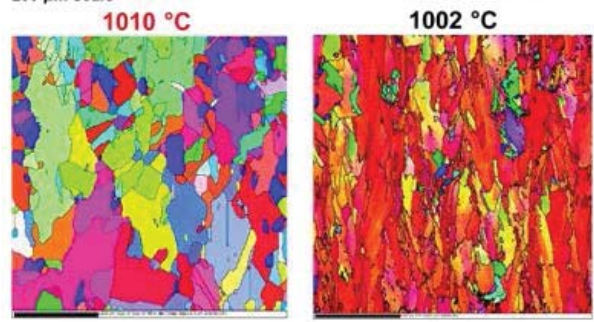

$1038^{\circ} \mathrm{C}$
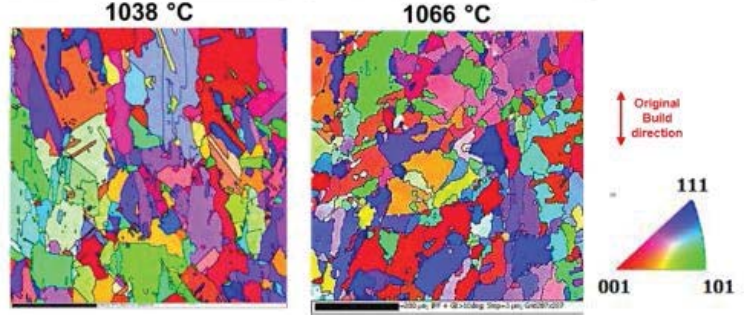

Figure 16. Wide-field EBSD maps for all tested stress relief temperature conditions. Color is based on inverse pole figure (IPF) of crystal directions oriented in the original build direction (oriented vertical) - IPF legend in lower right corner.

Figure 16 presents all wide-field EBSD maps for the six stress relief conditions analyzed. Maps are colored by the orientation of crystal directions (inverse pole figure) relative to the build direction - for example, red color indicates that the $\langle 100\rangle$ crystal direction is oriented parallel to the build direction. Black lines indicate grain boundaries (defined as $>15^{\circ}$ misorientation).

The as-built structure has a strong $\{001\}<100>$ fiber texture ("cube" texture) in the build direction, with a strongly aligned columnar grain structure. This textured and columnar structure is largely maintained after $1750^{\circ} \mathrm{F}$ and $1800^{\circ} \mathrm{F}$ stress relief treatments, though there is evidence of minor amounts of recrystallization that breaks up the texture. These structures are partially recrystallized. Significant variance in intragranular orientation is observed in these samples, and the patterning quality is low (evidenced by large number of unpatterned points and are mapped as white).
Stress relieving at $1850^{\circ} \mathrm{F}$ and above results in complete recrystallization of the material. All evidence of prior texture and columnar structure is replaced by randomly oriented, nominally equiaxed grains with a significant presence of annealing twins. Patterning quality is greatly improved in these samples (indicative of low lattice strain), and intragranular orientation variance appears low. Based on this analysis, the $1.5 \mathrm{hr}$ recrystallization temperature for $718 \mathrm{Plus}$ processed by DMLS is around $1850^{\circ} \mathrm{F}$. The presence of partial recrystallization at $1750^{\circ} \mathrm{F}$ and $1800^{\circ} \mathrm{F}$ suggests that it may be feasible to achieve full recrystallization at a reduced temperature if the stress relief cycle is extended beyond the current duration of 1.5 hours.

A key goal of the EBSD analysis is to determine the grain size of as a function of stress relief temperature. Grain size is monitored by the mean linear intercept method (ASTM E112), measured in the direction transverse to the original build direction, presented in Figure 17. The starting grain size is $\sim 20 \mu \mathrm{m}$ in the as-built condition. Upon stress relieving at $950{ }^{\circ} \mathrm{C}$ and $980^{\circ} \mathrm{C}$ the measured grain size is refined, due to the incidence of partial recrystallization (fine recrystallized nuclei pull the average measurement down, even though the overall structure appears to be unchanged). The grain size is marginally increased upon complete recrystallization at $1010{ }^{\circ} \mathrm{C}$, and subsequent increases in stress relief temperature result in progressively increasing grain growth. This analysis indicates that the finest as-stress-relieved grain size can be obtained by optimizing the stress relief condition to the minimum temperature necessary to achieve full recrystallization.

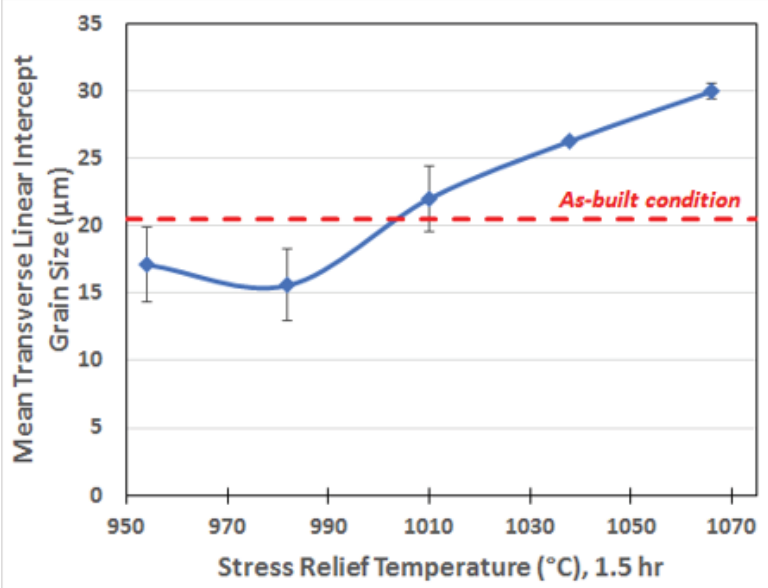

Figure 17. Mean linear intercept grain size for each stress relief condition. Intercept length measured from wide-field EBSD maps in Figure 1 in the orientation transverse to the build direction. Analysis includes twin boundaries as grain boundaries.

To quantify microstrain, band contrast is calculated for each diffraction pattern collected throughout the wide-field EBSD maps in Figure 16, presented in Figure 18. The most significant shift (particularly in the distribution width rather than the mean) occurs between $980{ }^{\circ} \mathrm{C}$ and $1010{ }^{\circ} \mathrm{C}$, where recrystallization greatly enhances the pattern quality, attributed to the dramatic reduction in residual microstrain. 

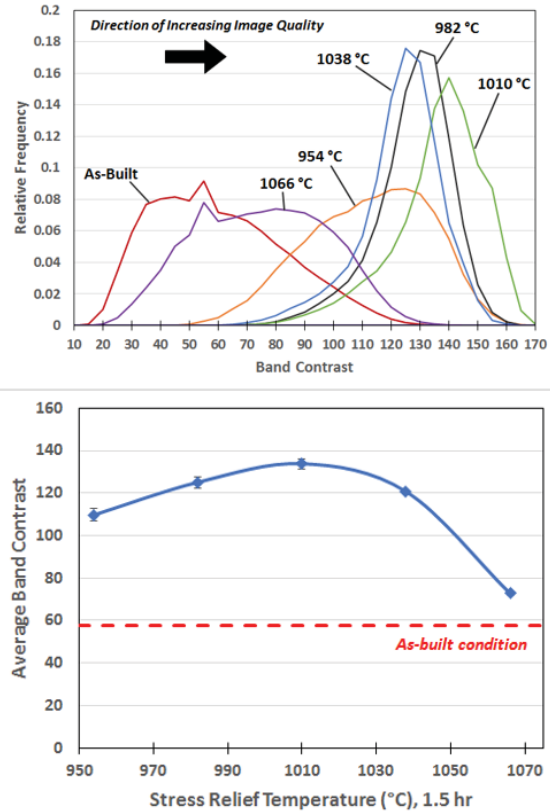

Figure 18. Band contrast measurements from wide-field EBSD maps (Figure 1) for each stress relief condition. Top: distribution in band contrast for all points within each EBSD map; Bottom: Mean band contrast averaged over entire map of each condition.

The second metric used to quantify microstrain is the Mean Intragranular Misorientation (MIM). For each grain within the EBSD maps contained in Figure 16, the average misorientation from the mean grain orientation is calculated from the raw diffraction data. This metric is calculated on an intragranular basis to exclude the misorientations associated with grain boundaries (high-misorientation boundaries that are not associated with microstrain in the crystal lattice). An MIM value was calculated for each individual grain within the EBSD map, yielding a statistical spread in MIM over hundreds of grains. This metric is then averaged over all grains in each map to provide an overall MIM as a function of stress relief condition.

The statistical spread in MIM per condition, and the calculated overall MIM as a function of stress relief condition are plotted in Figure 19. This analysis indicates a significant drop in both the MIM distribution and overall MIM upon recrystallization at $1850^{\circ} \mathrm{F}$ and above. Though a small decrease in both MIM distribution and overall MIM is observed with partial recrystallization at $950{ }^{\circ} \mathrm{C}$ and $980{ }^{\circ} \mathrm{C}$, the most notable change is at the recrystallization event of $1010^{\circ} \mathrm{C}$. This trend indicates that full recrystallization is necessary to relieve residual microstrain, and that increasing temperature above the minimum necessary for recrystallization does not appreciably reduce residual microstrain. The mean intragranular misorientation (MIM) for all grains was extracted from each of these maps in the same manner previously described for the wide-field maps, plotted in Figure 19. As observed in the wide-field maps, a significant drop in MIM is observed upon recrystallization at $1010{ }^{\circ} \mathrm{C}$. Beyond this temperature the MIM does not change. These higher-integrity results reinforce the original conclusion that full stress relief occurs upon recrystallization, and that increasing temperature above that necessary for recrystallization does not benefit microstrain relaxation appreciably.
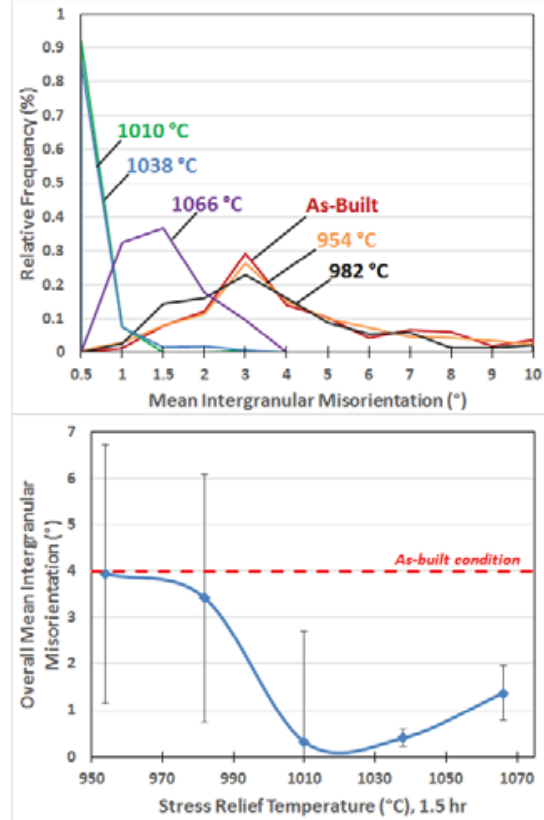

Figure 19. Mean Intragranular Misorientation (MIM) calculated from wide-field EBSD maps (Figure 1) for each stress relief condition. Top: distribution in MIM for all grains within each EBSD map; Bottom: Overall MIM averaged over all grains within each EBSD map.

Through EBSD, it was determined that the optimum 1 hour stress relief temperature for additively manufactured 718Plus is around $1010{ }^{\circ} \mathrm{C}$, which gives the finest grain structure and the minimum temperature necessary for recrystallization. Microstrain analysis confirmed that the minimum temperature necessary for recrystallization also minimized the strain left in the lattice.

Using further EBSD data gathered after varying annealing temperatures and times, a Johnson-Mehl-Avrami-Kolmogrov (JMAK) model [15] is fit to the time dependence of the recrystallized fraction, Y:

$$
Y=1-\exp \left(-K t^{n}\right)
$$

A best fit with an exponent of $\mathrm{n}=3.2$ is presented in Figure 20 . From these preliminary data, it is estimated that the optimum condition for efficient recrystallization with minimum temperature (finest grain size) is $990{ }^{\circ} \mathrm{C}\left(1815^{\circ} \mathrm{F}\right)$ for 6 hours.

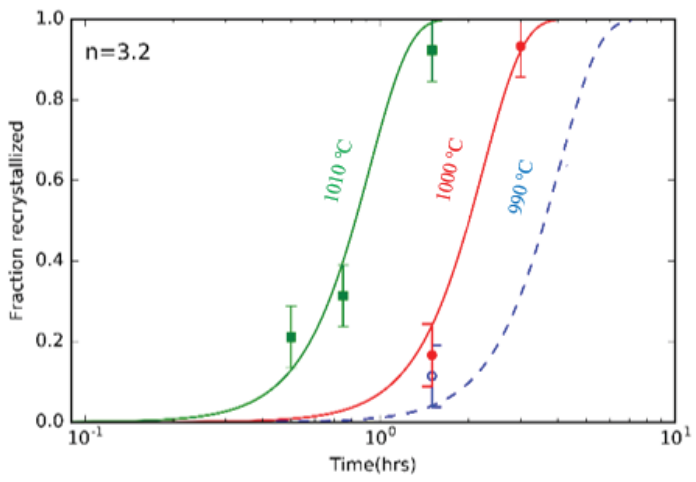

Figure 20. JMAK model for isothermal recrystallization. 


\section{Probabilistic Sensitivity Analysis}

A probabilistic sensitivity analysis was performed on the yield strength model to address two objectives. First, to quantify variability and/or uncertainty in predicted yield strength based on preliminary input random variable definitions, and second to quantify the relative impacts of the input random variables on yield strength. Probabilistic analysis was used to predict the cumulative distribution function (CDF) of yield strength as well as probabilistic sensitivities by propagating the input random variables through the model. The approach involved developing a fast-running Gaussian Process surrogate model for the QuesTek AIM yield strength model in order to facilitate Monte Carlo analysis.

First, based on discussions with the model developers, seven key input random variables were identified, as listed in Table II. These variables are expected to have a strong influence on yield strength and/or are subject to significant variability or uncertainty. Each variable was assigned a mean and standard deviation, as shown in Table II. The mean represents a nominal value, and the standard deviation represents the amount of scatter or uncertainty. These values are based on expected material processing ranges, historical data, and expert opinion. For the purposes of the probabilistic analysis, each variable is treated as a Gaussian random variable. Random variable definitions are preliminary and are used to gauge the general impact of input variation and/or uncertainty on predicted yield strength.

Table II. Preliminary input random variable definitions for yield strength model

\begin{tabular}{|l|l|l|}
\hline Variable & Mean & Std. Dev. \\
\hline Solution treatment temperature $\left({ }^{\circ} \mathrm{C}\right)$ & 968 & 4.63 \\
\hline Cooling rate $\left({ }^{\circ} \mathrm{C} / \mathrm{s}\right)$ & 2.2 & 0.56 \\
\hline Age 1 temperature $\left({ }^{\circ} \mathrm{C}\right)$ & 788 & 2.78 \\
\hline Age 2 temperature $\left({ }^{\circ} \mathrm{C}\right)$ & 704 & 2.78 \\
\hline Grain size $($ micron $)$ & 60 & 6.67 \\
\hline Interfacial energy $\left(\mathrm{J} / \mathrm{m}^{2}\right)$ & 0.028 & 0.00133 \\
\hline Gamma-prime APB energy $\left(\mathrm{J} / \mathrm{m}^{2}\right)$ & 0.225 & 0.005 \\
\hline HP-coefficient $\left(\mathrm{MPa}-\right.$ micron $\left.^{2}\right)$ & 0.682 & 0.0167 \\
\hline
\end{tabular}

Next, a design of computer experiments was generated with 200 cases over the 8 variables. A uniform Latin Hypercube design [16] was used, in which the lower and upper bound for each variable was defined as the mean $+/$ - three standard deviations. The yield strength corresponding to each of these 200 cases was computed using the QuesTek AIM model. The NESSUS ${ }^{\circledR}$ software was then used to fit the resulting data to a Gaussian Process response surface model [17] with a squared-exponential correlation function, in which the parameters were estimated via maximum likelihood. The goodness of fit of the response surface model was assessed using leave-one-out cross-validation [17]. The root-mean-squared-error is $6 \mathrm{MPa}(0.85 \mathrm{ksi})$, and the predictive $R^{2}$ value, based on the predictive error sum of squares, is 0.978 . This indicates that the response surface model captures most of the variation in the output and is suitable for the sensitivity anaylsis, although future work may consider increasing the number of training points in order to improve the model accuracy.

The response surface model was then used to conduct a parameter variation study, in which the parameters were varied one at a time, with the results shown in Figure 21. The parameter variation results give an indication of the nature of the influence of the eight variables on the model output.

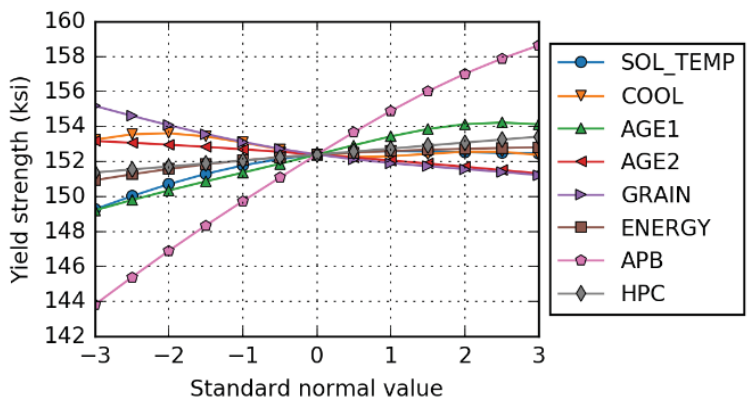

Figure 21. Parameter variation results based on yield strength response surface model. The standard normal value represents the number of standard deviations from the mean.

Finally, the NESSUS software was used to exercise the response surface model probabilistically, based on the input definitions in Table II. A Monte Carlo analysis provided probabilistic sensitivity results, which are normalized versions of the derivatives of probability with respect to the mean and standard deviation of each input. The probabilistic sensitivity results are computed for each probability level on the CDF and indicate how sensitive the predicted probability is to the mean and standard deviations that are defined in Table II. The probabilistic sensitivities computed at the lower tail of the $\mathrm{CDF}(\mathrm{u}=-3.2)$ are shown in Figure 22, and similar trends were seen at other points along the CDF. These results confirm that the gamma-prime APB energy is the biggest driver in yield strength uncertainty.

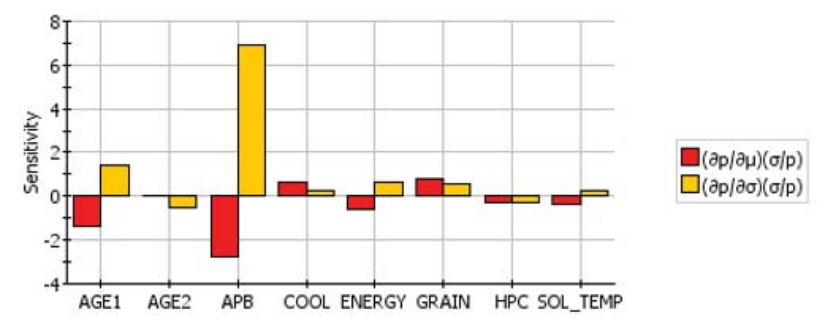

Figure 22. Probabilistic sensitivities for yield strength with respect to the mean and standard deviation of each input random variable.

\section{Conclusions}

In summary, the DARPA OM program drove several major advancements. A quantitative understanding of the 718Plus microstructure after extremely high cooling rates was gained through optical and electron microscopy. The effect of these novel microstructures on the previously standard heat treatment was also studied, leading to a greater understanding of recrystallization and microstrain interactions in the additively manufactured structure. Tensile tests were run to translate the current yield strength models to a new alloy system at room temperature, and run at high temperature to extend the tensile models to a new temperature range. Empirical data was used to both calibrate and validate the microstructural models and strength models in different areas. With the greater quantification of the effects of the novel processing method on the microstructure and mechanical behavior of 718Plus, the program will continue to drive towards rapid qualification of aerospace components in the coming years. 
Advanced electron backscatter diffraction (EBSD) allowed assessment of the feasibility of optimizing the stress relief cycle for more refined grain size. A minimum grain size is achieved when the stress relief temperature is minimized to avoid grain growth, thus achieving higher component strength.

An analysis of the minimum temperature necessary for recrystallization during the stress relief cycle must be balanced against the degree to which residual strains are relieved during the treatment. EBSD microanalysis has provided direct measurement of both the degree and scale of recrystallization, and the residual microstrain distributions after annealing.

For further information regarding Honeywell's DARPA Open Manufacturing Program, please see [19].

\section{Acknowledgements}

This work was performed under the DARPA Open Manufacturing Program entitled "Rapid Low Cost Additive Manufacturing" contract number HR001-12-C-0037 to Honeywell International Inc. The authors acknowledge the financial support and the guidance of the DARPA Program manager, Mr. Mick Maher and Subject Matter Expert panel (B. Cowles, J. Margiotta, W.Roy \& J. Williams).

We would also like to acknowledge our colleagues who have helped with different aspects of the program. From Honeywell: B. Baughman, P. Kantzos, M. Kemp, S. Singh, B. Shula, G. Levesque. From ESI: N. N'Dri, H.-W. Mindt. From Sigma Labs: M. Cola. V. Dave. From Stratonics: J. Craig \& T. Wakeman

Please note that the views, opinions, and/or findings expressed are those of the authors and should not be interpreted as representing the official views or policies of the Department of Defense or the U.S. Government

\section{References}

1. D. R. Askeland, The Science and Engineering of Materials (PWS-Kent Publishing Co., 1989)

2. A. Wilkinson, "Quantitative deformation studies using electron back scatter patterns", Acta Metal Mater 39 (1991) 3047-3055.

3. E. Lehockey et.al, "Mapping residual plastic strain in materials using electron backscatter diffraction", Electron Backscatter Diffraction in Materials Science, ed. A. Schwarts (Kluwer Academic/Plenum Publishers, 2000) 247-264

4. S. Wright et.al, "A Review of Strain Analysis Using Electron Backscatter Diffraction," Microsc. Microanal. 17 (2011) 316329.

5. R. W. Kozar, A. Suzuki, W.W. Milligan, et al. "Strengthening Mechanisms in Polyrcrystalline Multimodal Nickel-Base Superalloys," Met. Mat. Trans. A. V 40A, July 2009.

6. H. A. Roth, C. L. Davis, and R. C. Thomson, "Modeling Solid Solution Strengthening in Nickel Alloys," Met. Mat. Trans. A 28.6 (1997): 1329-1335.
7. L.A. Gypen, and A. Deruyttere, "Multi-Component Solid Solution Hardening,” J. Mater. Sci. 12, (1977), p.1028.

8. Mishima, Yoshinao, et al. "Mechanical properties of Ni 3 Al with ternary addition of transition metal elements." Transactions of the Japan institute of metals 27.1 (1986): 4150.

9. Y. Mishima, S. Ochiai, , N. Hamao, M. Yodogawa, T. Suzuki, "Solid Solution Hardening of Nickel - Role of Transition Metal and B-subgroup Solutes," Trans. Jpn. Inst. Met. 27, (1986) p.656

10. B. Reppich, "Particle Strengthening," Materials Science and Technology Vol. 6 Plastic Deformation and Fracture of Materials, ed. Mughrabi Wiley, 1992.

11. B. Reppich, "Particle strengthening," Materials Science and Technology, Materials Science and Technology, vol. 6, ed. H. Cahn, (1993).

12. A. P. Miodownik and N. Saunders "The calculation of APB Energies in $\mathrm{Ll}_{2}$, Compounds Using a Thermodynamic Database," Applications of Thermodynamics in the Synthesis and Processing of Materials, ed. P. Nash and B. Sundman, (Warrendale, PA: TMS, 1995), 91.

13. M. Sluiter and Y. Kawazoe, "A study of the thermodynamics of segregation and partial order at (111) antiphase boundaries in Ni3Al," Philosophical Magazine A, 78, 1998, 1353-1364

14. W. W. Milligan, et al. "Effects of microstructure on the high temperature constitutive behavior of IN100." Superalloys 2004 (Warrendale, PA: TMS, 2004): 331-339.

15. P. N. Kalu, D. R. Waryoba, “A JMAK-microhardness model for quantifying the kinetics of restoration mechanisms in inhomogeneous microstructure”, Mat. Sci. Eng. A, 464, (2007) p 68-75

16. T. Santner, B. Williams, and W. Notz, The Design and Analysis of Computer Experiments (New York, NY: Springer Verlag, 2003).

17. J. Martin and T. Simpson. "Use of kriging models to approximate deterministic computer models." AIAA Journal, 43 (4) (2005), 853-863

18. B.H. Thacker, et al. "Probabilistic engineering analysis using the NESSUS software." Structural Safety 28 (2006), 83-107.

19. A.D. Peralta, et al. "Towards Rapid Qualification of Powderbed Laser Additively Manufactured Parts," Materials and Manufacturing Innovation, IMMI. In-press. 